lems of stability. So the Indian team's work really represents a big advance. Clearly, rapid quenching near glassforming compositions can produce not just partially but fully crystalline materials with fine-scale microstructures.

Until recently, the focus of interest was on metallic glasses themselves, and materials with some crystallinity were regarded as rejects. The realization that their properties can be outstanding, and the link with the current interest in nanostructured materials, has changed all that. The new results of Nagarajan and Chattopadhyay show that the method can be applied to a wide range of particulate and matrix phases. The materials once thrown away by those looking for metallic glasses now prove that a previously unexploited processing route can produce nanometre-scale microstructures and exciting properties.

A. Lindsay Greer, of the Department of Materials Science and Metallurgy, University of Cambridge, UK, is currently on leave at the Section d'Études de la Solidification, CEREM-DEM Centre d'Études Nucléaires de Grenoble, 85X, 38041 Grenoble cedex. France.

\title{
Curling in the heat
}

DavidA. King

AN ingenious miniaturized heat sensor, shown here, has been developed by Jim Gimzewski and colleagues at IBM Rüschlikon specifically for studies of surface reactions ${ }^{1}$. A spin-off of the scanning probe microscopes developed in the same laboratory by Nobel prize winners Rohrer and Binnig, the device is based on the

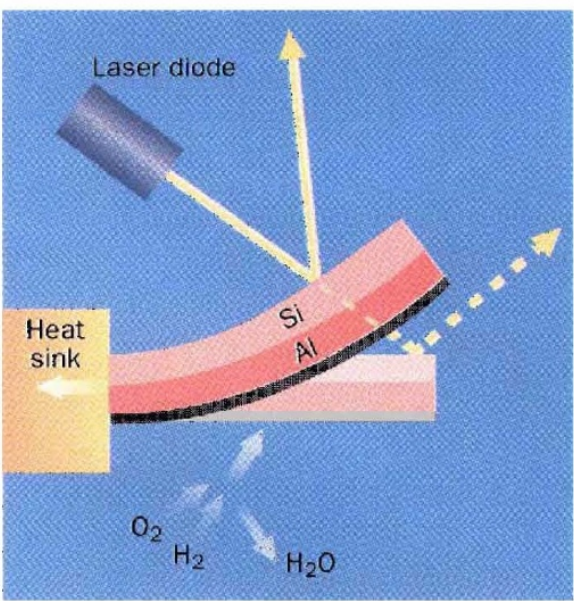

silicon levers used to detect atomic-scale protuberances on surfaces in the atomic force microscope.

The levers below are about $0.5 \mathrm{~mm}$ long and $1.5 \mu \mathrm{m}$ thick; each is a tiny sliver of silicon coated with a $0.4-\mu \mathrm{m}$ layer of aluminium. Smail changes in temperature cause the lever to bend because of the bimetallic effect: the thermal expansion coefficient of aluminium is eight times that of silicon. The degree of bending is proportional to the heat flux $\mathrm{d} Q / \mathrm{d} t$ absorbed by the lever, and is detected by measuring the change in direction of the reflected light beam, as shown schematically in the diagram. Radiation absorbed from the laser beam can itself be used as a means of calibrating the degree of bending by varying the laser power; a sensitivity of about $1 \AA$ per $n W$ absorbed radiation was obtained. In use as an atom probe, this type of lever detection system is capable of resolving movements of $0.1 \AA$, which indicates the extraordinary power sensitivity of the device.

To illustrate its potential as a micro-

\section{IMAGE UNAVAILABLE FOR COPYRIGHT REASONS}

Top, measuring the bending of a bilayer lever caused by heat released in reaction on a platinum surface (grey). Bottom, an array of 10 such sensors. calorimeter for surface processes, the authors deposited a thin $(0.04 \mu \mathrm{m})$ platinum film onto the lever, making a $\mathrm{Si}-\mathrm{Al}-\mathrm{Pt}$ sandwich. Under ultrahigh vacuum conditions, and starting with a clean platinum surface, the lever should bend when a dose of a reactive gas, such as $\mathrm{O}_{2}$, is introduced, because heat will be released in the exothermic process of chemisorption. Once adsorption is complete, the lever should return to its original position.

In their preliminary experiments, the vacuum was rather too poor for this to be done. Instead, a mixture of $\mathrm{O}_{2}$ and $\mathrm{H}_{2}$ was introduced into the reaction vessel, and the lever deflection recorded. Oscillations were observed, with a period of about 60 $\mathrm{s}$, and attributed to the existence of selfsustaining chemical oscillations which have previously been reported for the $\mathrm{H}_{2} \mathrm{O}$ formation reaction over platinum surfaces.

Clearly, one can foresee numerous applications for the device, perhaps in gas sensors, radiation detectors or quantitative calorimetry. A few years ago, I was one of a team who developed a quantitative microcalorimeter for studying the reactions between gases and single-crystal surfaces ${ }^{2-4}$. How does the new instrument compare? Our sensitivity is currently about $0.1 \mathrm{~nJ}$ (ref. 5) over a single-crystal sample area of $1.2 \times 10^{-5} \mathrm{~m}^{2}$; the sandwich lever system is, say the authors, sensitive to about $1 \mathrm{pJ}$ over a sample area of $1.4 \times$ $10^{-8} \mathrm{~m}^{2}$.

Expressed as a sensitivity per unit area, there is not much to choose between them. But clearly the different sample sizes of the two instruments give rise to different advantages and disadvantages. The principal disadvantage of the smaller sample size is the severe difficulty in measuring the gas sticking probability, and hence the amount of gas adsorption, a problem readily surmounted in our system $^{2}$. A major advantage could be in differential calorimetry studies, with one lever calibrated against a second lever without a reactive coating, of heat changes due to phase transitions in adsorbed layers and at clean surfaces. One problem which will have to be dealt with is the influence of these changes on the surface stress at the reactive surface: this will also cause the lever to bend. Nevertheless, this clever device looks likely to resurface in a wide range of applications.

David A. King is in the Department of Chemistry, University of Cambridge, Cambridge CB2 1EW, UK.

1. Gimzewski.J K Gerber Ch Meyer E \& Schittier R. R. Chem. Phys. Lett. 217. 589-594 (1994).

2. Borroni-Bird. C. E. \& King, D. A. Rev. scient. Instrum. 62. 2177-2185 (1991).

3. Yates, J. T. Jr Nature 360, 211-212 (1992).

4. Ai-Sarraf, N. Stuckless, J. T. \& King, D. A. Nature 360 243-245 (1992).

5. Stuckiess, T. Al-Sarraf, N. Wartnaby, C. \& King. D. A. J. chem. Phys. 99, 2202-2212 (1993). 